\title{
Images of unclassified and supergiant $B[e]$ stars disks with interferometry
}

\author{
Florentin Millour ${ }^{1,2}$, Anthony Meilland ${ }^{1}$, Olivier Chesneau ${ }^{2}$, Marcelo \\ Borges Fernandes ${ }^{3}$, Jose H. Groh ${ }^{1}$, Thomas Driebe ${ }^{1,4}$, Adrianne \\ Liermann $^{1}$ and Gerd Weigelt ${ }^{1}$ \\ ${ }^{1}$ Max-Planck Institute for Radioastronomy, auf dem Hügel 69, 53121 Bonn, Germany \\ email: fmillour@oca.eu \\ ${ }^{2}$ Observatoire de la côte d'Azur, Bd. de l'Observatoire, 06304 Nice, France \\ ${ }^{3}$ Observatorio Nacional, Rua General José Cristino, 77, Rio de Janeiro, Brazil \\ ${ }^{4}$ German Aerospace Center (DLR), Königswinterer Str. 522-524, 53227 Bonn, Germany
}

\begin{abstract}
B $[\mathrm{e}]$ stars are among the most peculiar objects in the sky. This spectral type, characterised by allowed and forbidden emission lines, and a large infrared excess, does not represent an homogenous class of objects, but instead, a mix of stellar bodies seen in all evolutionary status. Among them, one can find Herbig stars, planetary nebulae central stars, interacting binaries, supermassive stars, and even "unclassified" B [e] stars: systems sharing properties of several of the above. Interferometry, by resolving the innermost regions of these stellar systems, enables us to reveal the true nature of these peculiar stars among the peculiar $\mathrm{B}[\mathrm{e}]$ stars.
\end{abstract}

Keywords. techniques: high angular resolution, techniques: interferometric, binaries (including multiple): close, stars: individual (HD 87643, HD 62623), stars: mass loss, stars: winds, outflows

\section{Introduction}

We started an observing program covering the brightest unclassified and (candidate) supergiant B $[\mathrm{e}]$ stars, by using the Very Large Telescope Interferometer. For now, about 10 targets have been observed using a combination of AMBER (near-IR) and MIDI (midIR) observations. Here we focus on imaging and therefore stick to AMBER observations of two of the targets: the unclassified B[e] star HD87643 and the supergiant A[e] star HD 62623.

\section{HD 87643}

We used an extensive dataset spanning several orders of magnitude of spatial resolution to partly unveil the nature of this stunning system.

Wide-field images: Many new details are seen in our wide field image of the nebula around HD87643: blown up structures in the large scale nebula, which could be the result of an eruption much like in LBVs, and a series of arcs, unseen before, which could be the result of periodic ejections of matter.

Interferometric images: Our interferometric images, made using the AMBER/VLTI instrument, show a companion star to the bright star. In addition, the primary star exhibits an extended shell ( 4 mas), and background emission is detected.

Interpretation: Using a model involving both stellar components plus a resolved background, we were able to separate individual spectra in $\mathrm{H}, \mathrm{K}$ and $\mathrm{N}$ bands. We find that the primary star is enshrouded in a dust shell heated at the dust-sublimation temperature: we resolved the inner region of the HD87643 disk. The secondary component 

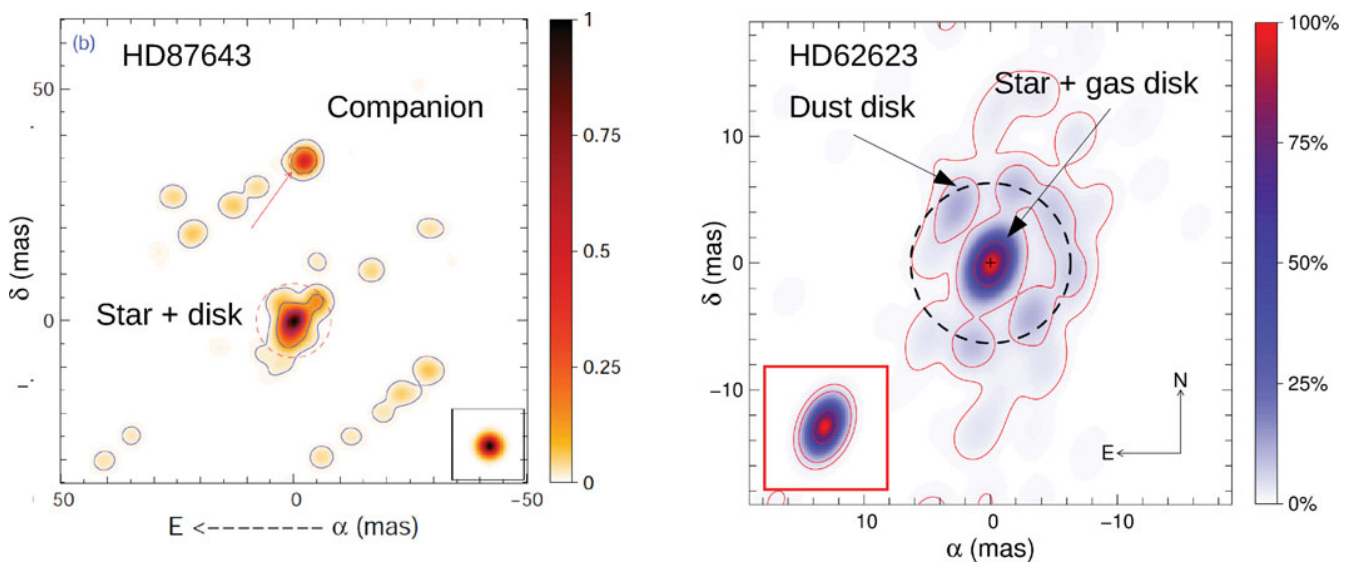

Figure 1. Left: AMBER/VLTI image of the star HD87643, revealing a companion star and a disk around the primary star. Right: AMBER/VLTI image of the previously-putative disk around the star HD62623. A spectrally-resolved image-cube could be synthesised, evidencing Keplerien rotation in this disk.

is also enshrouded in dust, at temperatures ranging from 500 to $50 \mathrm{~K}$. The resolved background holds most of the silicate emission, hence being likely composed of colder condensed dust. As a conclusion, and since the distance to the system is still poorly constrained, HD87643 could be a YSO instead of an evolved object. See Millour et al. 2009 for more details.

\section{HD 62623}

This star was already extensively studied by spectroscopy Plets et al. 1995. Hence it was already known that it hosted a circumstellar disk.

The continuum: We clearly resolve the gas and the dust disk in the continuum, imaging for the first time the disk of an evolved star. The image shows an outer ring corresponding to the inner rim where dust sublimation occurs, while we also resolve a central region where free-free emission from the gas takes place. The dust emission is asymmetric, which we attribute to an inclination effect. This direct picture strikingly confirms what was indirectly inferred from spectroscopic data previously.

The $\mathrm{Br} \gamma$ line: Our high-spectral resolution image cube in the $\mathrm{Br} \gamma$ line directly shows the motion of the gas in different velocity bins. The combination of a model of the dust, which gives the on-sky orientation and inclination of the disk, and of a model of the rotating gas, allows us to claim that we detected Keplerian rotation in that disk. We can now clearly rule out expanding wind models for HD 62623. Instead, we have a Keplerian rotating disk, where expansion is negligible. Such a disk, unexpected for massive stars, is common in young stellar objects like Herbig stars. In the case of HD 62623, the presence of a very close unseen companion, previously detected by radial velocities, might be the key to the formation of such a rotating disk.

\section{References}

Millour, F., Chesneau, O., Borges Fernandes, M., Meilland, A. et al. 2009, A\&\&A, 507, 317

Plets, H., Waelkens, C. \& Trams, N. R. 1995, A\&A, 293, 363 